\title{
Generation of ultrafast electrons by simultaneous stimulated Raman backward and forward scattering
}

\author{
P. Bertrand, ${ }^{1}$ A. Ghizzo, ${ }^{1}$ S. J. Karttunen, ${ }^{2}$ T. J. H. Pättikangas, ${ }^{2}$ \\ R. R. E. Salomaa, ${ }^{3}$ and M. Shoucri ${ }^{4}$ \\ ${ }^{1}$ Université de Nancy-I, Laboratoire de Physique des Milieux Ionises, Bồte Postale 239, 54506 Vandœuvre, France \\ ${ }^{2}$ Nuclear Engineering Laboratory, Technical Research Centre of Finland, Espoo, Finland \\ ${ }^{3}$ Department of Technical Physics, Helsinki University of Technology, Espoo, Finland \\ ${ }^{4}$ Centre Canadien de Fusion Magnétique, Tokamak de Varennes, Varennes, Québec, Canada
}

(Received 18 June 1993)

\begin{abstract}
Fast electron generation in stimulated Raman forward and backward scattering is investigated. The simultaneous forward and backward scattering is shown to create an electrostatic field structure which is exceptionally efficient in producing highly relativistic electrons. The Vlasov-Maxwell simulations reveal that the two plasma wave packets generated by the backward and forward scattering are spatially separated, and thus form a two-staged electron "accelerator." The bulk electrons are accelerated from the lower boundary of the trapping region of the slower wave to the upper boundary of the trapping region of the faster wave. The mechanism of the electron acceleration is analyzed by Vlasov-Maxwell simulations with self-consistent fields and by test particle calculations with prescribed electrostatic fields.
\end{abstract}

PACS number(s): $52.35 . \mathrm{Mw}, 52.40 . \mathrm{Nk}$

Stimulated Raman scattering (SRS) is the parametric instability where an electromagnetic pump wave decays into a scattered wave and a plasma wave. In long uniform plasmas, the electrostatic fields may reach large amplitudes, and thus they can trap and accelerate electrons to high energies. The nonlinear wave-particle interactions and stochastic behavior of electrons play a very important role, for instance, in laser fusion, in radiofrequency heating and current drive, and in accelerator physics. In laser fusion, even a small number of energetic electrons can cause severe preheating and degrade the gain of the fusion target [1]. In magnetic fusion, it was recently suggested that the so-called beat-wave process [2] or SRS [3] in the microwave region could be used for current drive in tokamaks. In this application, the fast electrons are beneficial because they are almost collisionless, which improves the current drive efficiency.

In SRS, the scattered electromagnetic wave may propagate in backward or forward direction with respect to the pump wave. In both Raman forward (SRSF) and backward scattering (SRSB), the electron plasma waves travel forwards but their phase velocities may differ considerably. Usually in laser-plasma experiments, SRSB dominates over SRSF because of its larger gain. In underdense high-temperature plasmas, however, the Landau damping strongly limits SRSB, which makes possible the growth of the forward scattering [1]. The parameters corresponding to reactor-grade laser-plasma experiments and to SRS current drive in tokamak fusion reactors lie in the intermediate region where both processes can occur simultaneously $[4,5]$.

A simple model [4] based on coupled mode theory predicts that in a homogeneous plasma slab, SRSB and SRSF are localized in spatially distinct regions: SRSB in the front and SRSF in the rear part of the slab. There- fore, the corresponding plasma waves are also localized in these regions forming two wave packets with different phase velocities.

The phase velocity of the plasma wave created in SRSB is lower and closer to the electron thermal velocity than the phase velocity of the SRSF plasma wave. The intense SRSB plasma wave can interact with a fairly large number of bulk electrons which get trapped by the wave potential. If the amplitude of the SRSF plasma wave is so large that the trapping regions of the two plasma waves overlap in velocity space, numerous ultrafast electrons may be created. The role of SRSB is to extract bulk electrons, and the role of SRSF is to give them a further energy boost. Note that the two wave packets can be spatially separate-the overlapping of the trapping regions has to occur only in the velocity space.

It has previously been shown that even a single largeamplitude wave packet can accelerate and decelerate traversing electrons in a stochastic manner [6,7]. The behavior of the electrons can also be bistable: they either get a large velocity increment from the wave or no velocity increment at all. The motion of the electrons in the potentials of two spatially overlapping modes is even more complicated, and shows all the versatile features of chaos $[8,9]$. In two spatially separate wave packets, the stochastic motion of electrons has not, to our knowledge, been previously examined.

In this paper, we investigate the above idea of fast electron generation by Vlasov and test particle simulations. The Vlasov simulations demonstrate the localization of SRSB and SRSF in different regions of the plasma, and reveal the generation of a large amount of ultrafast electrons. The accurate simulations make it possible to follow in detail the motion of the electrons through the potentials of the two electrostatic waves. The strongly non- 
linear nature of the two-stage acceleration brings about unexpected features in the fast electron dynamics. Test particle simulations with prescribed electrostatic wave packets give further insight into the acceleration process.

Our simulation computer code $[10,11]$ is a nonperiodic Eulerian Vlasov code which assumes a plasma slab of a finite length. The code solves the relativistic Vlasov and Maxwell equations for plane waves propagating in a onedimensional electron plasma with a fixed ion background. The noiseless Eulerian code makes possible precise diagnostics of the electric fields, allowing accurate calculation of the envelopes and phases of the different modes.

For simplicity, we consider a homogeneous plasma slab of length $L$ surrounded by a void where an electromagnetic pump wave is launched at $x=0$. The length of the system is $L=64 \lambda_{0}=188 c / \omega_{p}$, where $\lambda_{0}$ is the pump wavelength in plasma. The frequency of the pump wave is $\omega_{0}=2.36 \omega_{p}$ (i.e., $n / n_{\mathrm{cr}}=0.18$ ), and the amplitude is $v_{0} / c=0.1$, where $v_{0}$ is the quiver velocity. The initial electron distribution function is Maxwellian with a temperature of $T_{e}=10 \mathrm{keV}$. These parameters are in the optimum operating region for simultaneous SRSB and SRSF [4].

The frequencies and wave numbers of the scattered waves can be estimated from the phase-matching conditions and the dispersion relations. If the plasma waves are assumed to obey the Bohm-Gross relation, their wave numbers are $k_{F} \lambda_{D}=0.18$ and $k_{B} \lambda_{D}=0.38$. The linear Landau damping of the plasma wave excited by SRSB is strong, $\Gamma_{B} / \omega_{p}=0.049$, in contrast to the wave excited by SRSF which is almost undamped $\left(\Gamma_{F} / \omega_{p}=3.5 \times 10^{-6}\right)$. The phase velocities (momenta) of the plasma waves are $v_{\mathrm{phB}}=0.45 c\left(p_{\mathrm{phB}}=0.50 m_{e} c\right)$ and $v_{\mathrm{phF}}=0.82 c$ $\left(p_{\mathrm{phF}}=1.42 m_{e} c\right)$

Figure 1 displays typical results of our Vlasov simulations at two different times. At the earlier time, $\omega_{p} t=180$, backward and forward scattering have grown to an appreciable level but the higher order nonlinear effects have not yet developed. In the total electro- static field of Fig. 1(a), the two main components have very clear-cut features because they are spatially separated and their wavelengths differ considerably. In the wave number spectra of Fig. 1(b), the corresponding two narrow peaks occur exactly at their expected positions for SRSB and SRSF. In Fig. 1(c), the action density envelopes of the electrostatic fields obtained by the Hilbert transform are shown. The electrostatic waves are spatially separated, the backward field extending from $x \omega_{p} / c=0$ to about 50, and the forward wave being peaked around $x \omega_{p} / c=90$. At time $\omega_{p} t=180$, the pump field, which is not shown, is still practically undepleted. Later at $\omega_{p} t=300$, the saturation effects and wave-wave and wave-particle interactions start to play a role.

In Fig. 2, we illustrate the fast electron generation by a phase-space plot and by two distribution functions corresponding to spatial averages over the regions $26.5 \leq x \omega_{p} / c \leq 50$ and $120.5 \leq x \omega_{p} / c \leq 144$, respectively. In the front part, $26.5 \leq x \omega_{p} / c \leq 50$, the dominant wave-particle interaction is due to the plasma wave created by SRSB. A plateau is formed in the distribution function (a) around $\gamma_{\mathrm{phB}}-1=0.11$, where $\gamma_{\mathrm{phB}}$ is the Lorentz factor at $v_{\mathrm{phB}}$. In the center of the plasma, represented by the distribution function (b), the plateau appears around $\gamma_{\mathrm{phF}}-1=0.75$. As the separatrices in the phase-space plot show, the trapping region extends from $\gamma-1=0.2$ to about 2.0, which agrees well with the observed plateau region. At the rear end of the plasma, the electrostatic field becomes weaker, and therefore the decoupled energetic electrons are streaming freely.

A key feature in the distribution functions in Fig. 2 is that the height of the plateau is only one order of magnitude smaller in the SRSF dominated region than in the SRSB region. This demonstrates that the efficiency of fast electron generation can be drastically increased when both the backward and the forward electrostatic fields are simultaneously present even though they are not spatially overlapping. We have also run simulations (a)
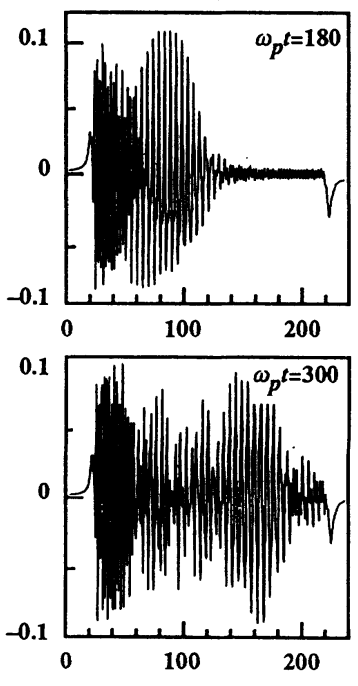

SPATIAL COORDINATE $x \omega_{p} / c$ (b)
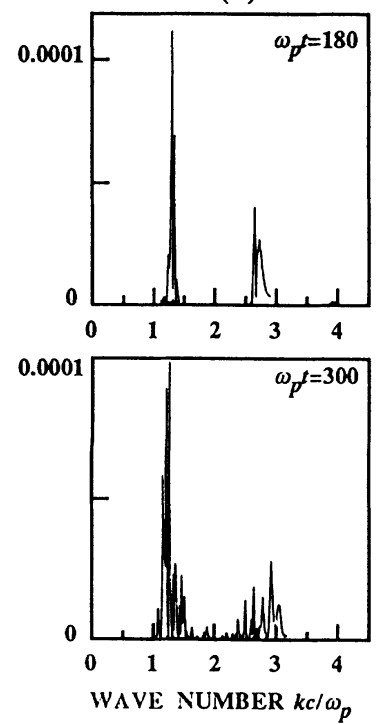

(c)
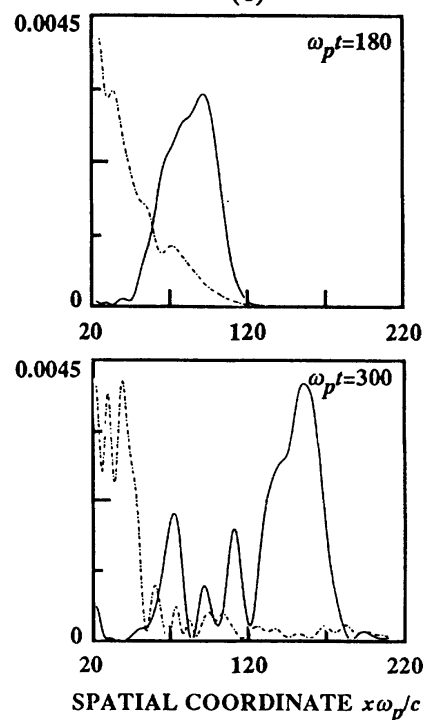

FIG. 1. Electrostatic fields at $\omega_{p} t=180$ (top) and $\omega_{p} t=300$ (lower frames). (a) Spatial dependence of the longitudinal electric field $e E / m_{e} c \omega_{p}$. (b) Wave number spectrum. (c) Spatial dependence of envelopes of the SRSF (solid line) and SRSB (dashed) plasma waves in action density units $\varepsilon_{0} \omega_{p}^{-1} \partial \epsilon / \partial \omega E^{2}$. 

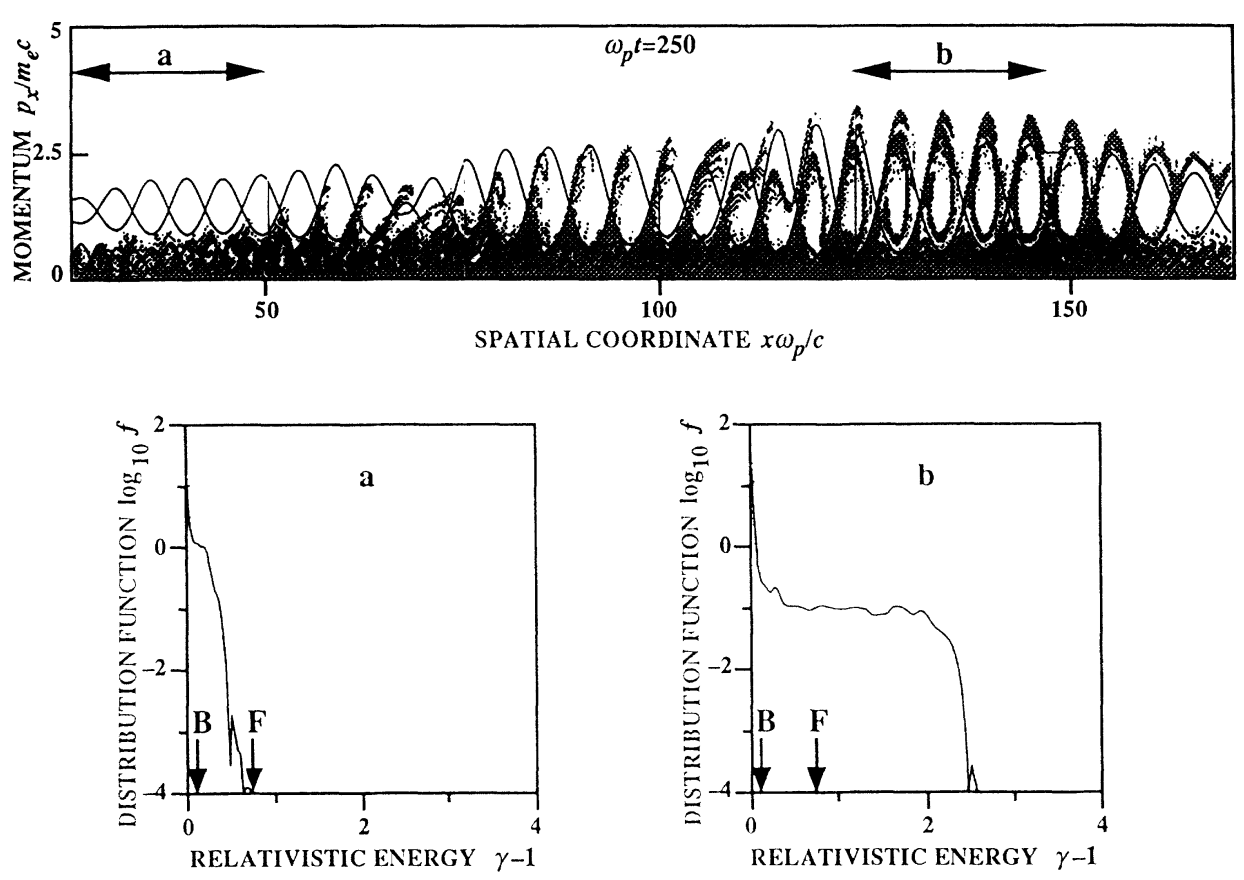

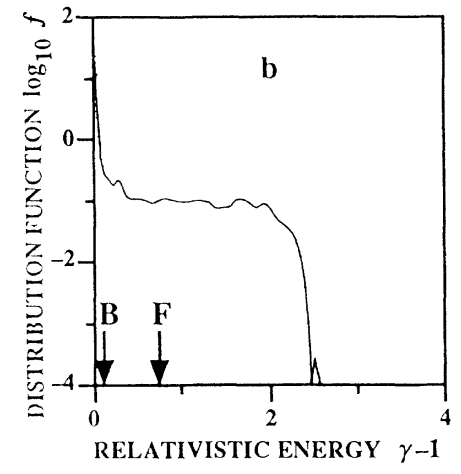

FIG. 2. Phase-space plot $(x-p)$ of the electron distribution function at $\omega_{p} t$ $=250$ (top). The solid line corresponds to the separatrix momentum of a marginally trapped electron. The markers $a$ and $b$ refer to the spatially averaged electron distribution functions shown in the lower frames as a function of $\gamma-1$. The arrows $(B, F)$ stand for the phase velocities of the plasma waves. where SRSB is suppressed due to a higher temperature. Then the height of the hot electron plateau was several orders of magnitude smaller than in Fig. 2, which clearly demonstrates the efficiency of simultaneous SRSB and SRSF in hot electron production.

To understand the behavior of individual electrons, we have studied the staged acceleration of electrons by test particle simulations in spatially separated fields which resemble the plasma waves generated by SRSB and SRSF. We have solved numerically the equations of motion of an electron in the two-component electrostatic field

$$
E=\sum_{i=F, B} E_{i}(x) \cos \left(k_{i} x-\omega_{i} t+\varphi_{i}\right)
$$

where Gaussian envelopes $E_{i}(x)=E_{i} \exp \left[-\left(x-x_{i}\right)^{2} / w_{i}^{2}\right]$ were assumed. The test electrons had the initial momenta $p_{\text {in }}$ at $x=0$, and the final momenta $p_{\text {out }}$ at $x=L$ were calculated. These calculations are analogous to those performed by Fuchs et al. [6] for a single nonrelativistic wave packet.

Inspired by the Vlasov simulations, we chose the two Gaussian fields centered at $x_{B}=L / 4$ and $x_{F}=3 L / 4$, where $L \omega_{p} / c=188$ was the length of the simulation box. The widths of the Gaussians were $w_{B, F} \omega_{p} / c=15$, and the amplitudes were $e E_{B, F} / m_{e} \omega_{p} c=0.1$ in accordance with those observed in the Vlasov simulations. For each calculated electron trajectory the field phases $\varphi_{i}$ were chosen randomly.

Figure 3 shows the final momenta $p_{\text {out }}$ of an ensemble of test electrons having an even distribution of initial momenta $p_{\text {in }}$. The crosslike structure in the lower left corner is due to the slower SRSB-like wave; the structure appearing at $p_{\text {in }} / m_{e} c>0.7$ is due to the faster SRSF-like wave. The differences in the SRSB and SRSF patterns arise from the relativistic effects dominating in SRSF.
The island of data points in the upper left-hand corner of Fig. 3 is due to the staged acceleration by the SRSB and SRSF plasma waves. It is quite remarkable that the electrons with $p_{\text {in }} / m_{e} c \approx 0.3$ are directly accelerated to $p_{\text {out }} / m_{e} c \approx 2-3$. SRSB alone would only produce electrons up to about $p_{\text {out }} / m_{e} c<1$. In thermal distribution at fusion temperatures, there is only a negligible amount of electrons with momenta $p_{\text {in }} / m_{e} c>0.7$ which are required for an efficient single-step acceleration by SRSF. Therefore, the synergy between SRSB and SRSF leads to an unanticipated amount of very fast electrons.

The homogeneous slab model of the plasma that was used in the Vlasov simulations has limitations. The model can be expected to describe well reactor-grade laser-plasma experiments where a very long homogeneous

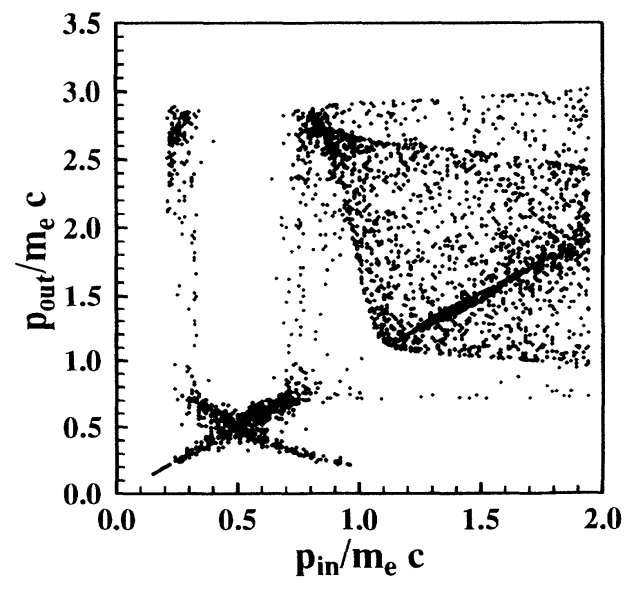

FIG. 3. Final momentum $p_{\text {out }} / m_{e} c$ as a function of incident momentum $p_{\text {in }} / m_{e} c$ for an ensemble of 2000 test electrons which propagate through two spatially separated Gaussian wave packets. 
plasma is formed. In SRS current drive, the interaction region near the magnetic axis is also well described by the homogeneous model. When the plasma has a weak density gradient, SRS occurs with a range of scattered frequencies. Density gradient also reduces the growth rates of both Raman processes, and therefore it affects the competition between SRSB and SRSF. A weak density gradient is not, however, expected to affect the spatial separation of SRSB and SRSF because the main reason for the separation is the wave propagation effect.

In the simulation model, other simplifications have also been made. The boundary conditions of a real plasma, for instance, in laser-plasma experiments are much more complicated than in our model. It should also be pointed out that the one-dimensional model overestimates to some extent the hot electron tail.

In conclusion, our Vlasov and test particle simulations show that simultaneous Raman backward and forward scattering are able to produce superhot electrons with $\mathrm{MeV}$ energies. The SRSB plasma wave preaccelerates a large number of electrons from the bulk of the distribution. They are then further energized by the faster SRSF plasma wave. An essential feature in this two-stage process is that SRSB and SRSF are spatially localized and separated. SRSB occurs near the front region, and SRSF grows much deeper inside the plasma slab. The simulations reveal how the bulk electrons are accelerated from the lower boundary of the trapping region of the slower electrostatic wave to the upper boundary of the trapping region of the faster wave. This is in contrast to spatially overlapping wave packets investigated earlier $[8,9]$, where electron behavior resembles more a diffusion process than a coherent two-stage acceleration. In the simulations, it is found that the effect is robust and easy to reproduce in the parameter region determined earlier [4] for simultaneous SRSB and SRSF.

The reactor-grade laser fusion experiments are in the parameter region where simultaneous Raman forward and backward scattering can occur. The possibility of simultaneous occurrence of SRSB and SRSF and the consequent hot electron production make the prevention of SRS particularly important. It is of utmost importance to prevent the preheating of the pellet core that would be caused by the enormous fast electron tail of Fig. 2 .

The two-stage acceleration of electrons described above can also have important applications to current drive in tokamaks. One has to reconsider the efficiency derived for SRS current drive $[2,3]$. It may also be possible to apply similar techniques to current drive with other radio frequency waves. One could use two different beams which are not spatially overlapping and have different phase velocities. In such an experiment, one could find a two-stage acceleration process similar to the one described above.

The authors are indebted to the Centre de Calcul Vectoriel pour la Recherche (Ecole Polytechnique, Palaiseau, France) for computer time allocation on the Cray-2. This research was partly supported by the Finnish Ministry of Trade and Industry.
[1] R. E. Turner, K. Estabrook, R. P. Drake, E. A. Williams, H. N. Kornblum, W. L. Kruer, and E. M. Campbell, Phys. Rev. Lett. 57, 1725 (1986).

[2] B. I. Cohen, R. H. Cohen, B. G. Logan, W. McCay Nevins, G. R. Smith, A. V. Kluge, and A. H. Kritz, Nucl. Fusion 28, 1519 (1988).

[3] S. J. Karttunen, T. J. H. Pättikangas, R. R. E. Salomaa, and S. K. Sipilä, Nucl. Fusion 31, 1079 (1991).

[4] S. J. Karttunen and R. R. E. Salomaa, Laser Part. Beams 10, 75 (1992).

[5] K. Estabrook and W. L. Kruer, Phys. Fluids 26, 1892 (1983).
[6] V. Fuchs, V. Krapchev, A. Ram, and A. Bers, Physica D 14, 141 (1985).

[7] D. L. Bruhwiler and J. R. Cary, Phys. Rev. Lett. 68, 255 (1992).

[8] B. V. Chirikov, Phys. Rep. 52, 263 (1979).

[9] A. B. Rechester and T. H. Stix, Phys. Rev. Lett. 36, 587 (1976).

[10] P. Bertrand, A. Ghizzo, S. J. Karttunen, T. J. H. Pättikangas, R. R. E. Salomaa, and M. Shoucri, Phys. Fluids B 4, 3590 (1992).

[11] P. Bertrand, A. Ghizzo, T. W. Johnston, M. Shoucri, E. Fijalkow, and M. R. Feix, Phys. Fluids B 2, 1028 (1990). 

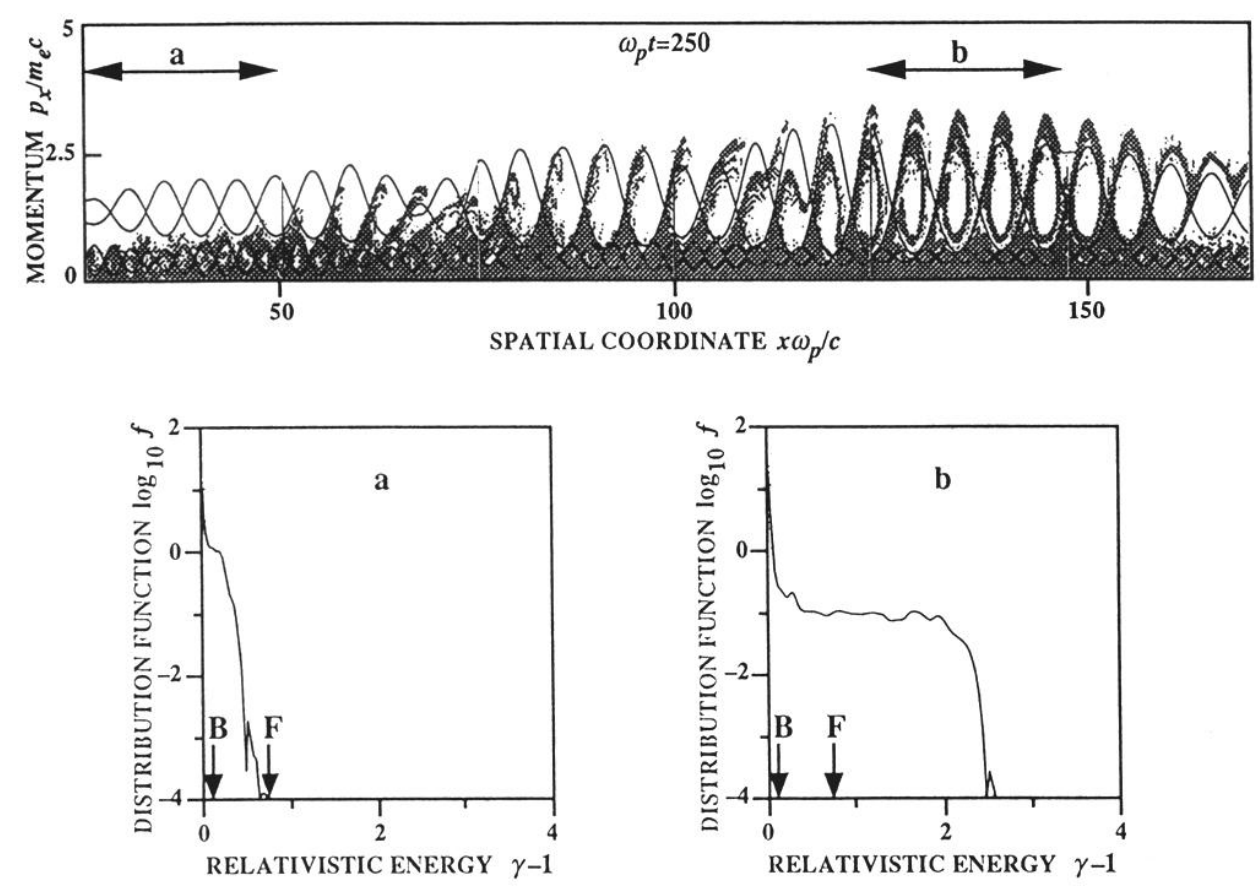

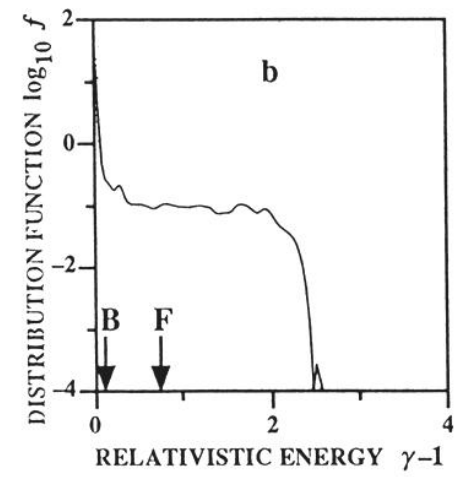

FIG. 2. Phase-space plot $(x-p)$ of the electron distribution function at $\omega_{p} t$ $=250$ (top). The solid line corresponds to the separatrix momentum of a marginally trapped electron. The markers $a$ and $b$ refer to the spatially averaged electron distribution functions shown in the lower frames as a function of $\gamma-1$. The arrows $(B, F)$ stand for the phase velocities of the plasma waves. 